\title{
Self-Synchronized Phenomena Generated in Coupled Nonlinear Self-Excited Oscillators with Stick-Slip Motion*
}

\author{
Tomoki ONO**, Takahiro KONDOU** and Yasuhiro BONKOBARA** \\ ${ }^{* *}$ Department of Mechanical Engineering, Faculty of Engineering, Kyushu University \\ 744 Motooka, Nishi-ku, Fukuoka-shi, Fukuoka, 819-0395 Japan \\ E-mail: ono_tomo@mvib.mech.kyushu-u.ac.jp
}

\begin{abstract}
A simple nonlinear system that generates synchronization is developed to clarify the mechanism behind the phenomenon. The present system consists of two nonlinear self-excited oscillators subjected to Coulomb friction, resulting in the generation of stick-slip motion. These oscillators are directly coupled in series by a coil spring and a dashpot. The synchronization generated in this system is studied both analytically and experimentally. The validity of the model and the numerical method based on the shooting method are verified by comparing the numerical and experimental results. As a result, it is found that two types of synchronized solutions exist in this system, and the vibratory patterns of the synchronized solutions are closely related to the undamped free vibration characteristics of the model without Coulomb friction. In addition, differences between the occurrence mechanisms of the synchronized solutions are analytically confirmed by examining the energy transmission between the two oscillators through the coupling element. It is also proved that unstable regions caused by internal resonance exist in the solution branch, in which the two oscillators vibrate nearly in phase.
\end{abstract}

Key words: Nonlinear Vibration, Self-Excited Vibration, Synchronization, Frictional Vibration, Shooting Method

\section{Introduction}

When some nonlinear self-excited oscillators with specific frequencies are coupled, all of the frequencies of the oscillators can be entrained into a certain frequency due to the mutual interaction between them ${ }^{(1)}$; this is widely known as synchronization. This is a nonlinear phenomenon and can occur in a wide range of interdisciplinary fields. For example, bioscientists have investigated the synchronization between heartbeats and respiratory organs and the synchronous firing of brain neurons ${ }^{(2)}$ to clarify their mechanisms and explore their potential applications. Meanwhile, mechanical engineers have studied its application to mechanical systems for a long time, and numerous practical examples (vibratory elevator, vibratory conveyor, etc.) have been developed ${ }^{(3)}$. In these instances, if synchronization were utilized, desirable motions could be efficiently realized in machinery without complicated controls. However, because the synchronization generated in nonlinear multi-degree-of-freedom systems is so complicated, its mechanism is not well understood; therefore, the machinery to which the phenomenon is applicable is limited.

Based on this situation, some of the current authors have investigated the synchronization generated in pendulum-type oscillators ${ }^{(4)}$ and rotor-type oscillators ${ }^{(5),(6)}$ to 
clarify the mechanism behind the synchronization. Some features of the stable synchronized solutions and the differences in the solution structures based on the nonlinearity of the oscillators were clarified. By comparing the synchronized solutions with the nonlinear normal modes generated in undamped nonlinear free vibration systems, a strong relationship between them was confirmed. In these models, however, the branching structures and the stability of the synchronized solutions tended to become complicated with increasing the degrees of freedom of the elements coupling the oscillators (the coupling elements). Hence, the essential synchronization characteristics are not well understood. This suggests that if the degrees of freedom of the coupling element were eliminated by directly coupling the oscillators using such mechanisms as coil springs, the phenomenon and the branching structures of the synchronized solutions would become simple, and then it would be easy to clarify the mechanism behind the synchronization.

In the present study, we develop a new model in which two oscillators are directly coupled using a coil spring and a dashpot. The oscillator is a single-degree-of-freedom mass-damper-spring system placed on a belt conveyor that is driven at a constant speed. Stick-slip motion (a kind of nonlinear self-excited vibration) is frequently caused by Coulomb friction acting on the interfaces between the belt surface and each of the oscillators. The present study analytically and experimentally investigates the characteristics of the synchronization generated in such a simple system and then studies the mechanism by examining the energy transmission between the oscillators through the coupling element.

\section{Fundamental equations}

\subsection{Analytical model}

Figure 1 shows the analytical model, which consists of two self-excited oscillators coupled using a coil spring (spring constant: $K$ ) and a dashpot (damping coefficient: $C$ ). The oscillator $i(i=1,2)$ is composed of a mass block (mass: $\left.m_{i}\right)$ connected to a fixed wall by a coil spring (spring constant: $k_{i}$ ) and a dashpot (damping coefficient: $c_{i}$ ) and is placed on a horizontal belt conveyor that is driven at a constant speed $V_{i}$. The Coulomb friction force inducing stick-slip motions acts on the interfaces between the belt surface and each of the blocks. The blocks of oscillator 1 and oscillator 2 are hereinafter referred to as Block 1 and Block 2, respectively. The present model is a two-degree-of-freedom system in which only oscillators have a degree of freedom and consists of the minimum number of elements required for synchronization generation. In addition, with respect to dynamics, if the viscous damping forces are neglected (since the influence is small), we only have to consider the restoring forces and the Coulomb friction. Therefore, the present model is one of the simplest models in which synchronization is generated and is suitable for investigating the mechanism.

\subsection{Equation of motion}

First, we consider the case in which both blocks are in the slip condition. The displacement $x_{i}$ of Block $i$ is defined as shown in Fig. 1. The equation of motion is given by

$$
m_{i} \ddot{x}_{i}+c_{i} \dot{x}_{i}+k_{i} x_{i}+C\left(\dot{x}_{i}-\dot{x}_{j}\right)+K\left(x_{i}-x_{j}\right)=\mu_{d i} m_{i} g,
$$

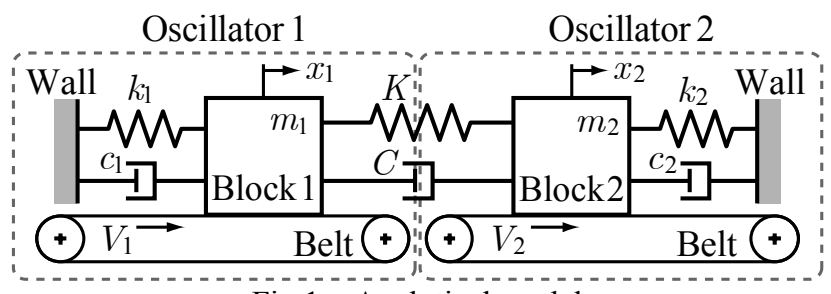

Fig.1 Analytical model 
where $" . "$ stands for $d / d t,(i, j)=(1,2)$ or $(2,1), g$ is the gravitational acceleration, and $\mu_{d i}$ is the coefficient of dynamic friction between the belt surface and Block $i$.

On the other hand, when Block $i$ is in the stick condition, the motion is expressed by

$\dot{x}_{i}=V_{i}, \quad \ddot{x}_{i}=0$.

Then, the static friction force $\tilde{f}_{s i}$ keeping Block $i$ resting against the belt is derived as Eq. (3) using Eqs. (1) and (2).

$$
\tilde{f}_{s i}=c_{i} V_{i}+k_{i} x_{i}+C\left(V_{i}-\dot{x}_{j}\right)+K\left(x_{i}-x_{j}\right)
$$

In the analysis, the following system parameters are introduced:

$$
\omega_{i}=\sqrt{\frac{k_{i}}{m_{i}}}, \quad \tilde{\omega}_{i}=\sqrt{\frac{K}{m_{i}}}, \quad \zeta_{i}=\frac{c_{i}}{2 \sqrt{m_{i} k_{i}}}, \quad \tilde{\zeta}_{i}=\frac{C}{2 \sqrt{m_{i} K}} .
$$

Rearrangement of Eqs. (1) and (3) by substituting the parameters yields

$$
\begin{aligned}
& \ddot{x}_{i}+2 \zeta_{i} \omega_{i} \dot{x}_{i}+\omega_{i}^{2} x_{i}+2 \tilde{\zeta}_{i} \tilde{\omega}_{i}\left(\dot{x}_{i}-\dot{x}_{j}\right)+\tilde{\omega}_{i}^{2}\left(x_{i}-x_{j}\right)=\mu_{d i} g, \\
& f_{s i}=2 \zeta_{i} \omega_{i} V_{i}+\omega_{i}^{2} x_{i}+2 \tilde{\zeta}_{i} \tilde{\omega}_{i}\left(V_{i}-\dot{x}_{j}\right)+\tilde{\omega}_{i}^{2}\left(x_{i}-x_{j}\right) .
\end{aligned}
$$

Equations (2), (5), and (6) are the fundamental equations for the present model.

\subsection{Switching condition of motion}

We must obtain the time at which the block motion switches (hereinafter referred to as the switching time) to analyze the stick-slip motion. This condition is described as follows.

First, if $\dot{x}_{i} \neq V_{i}$ at a certain moment, Block $i$ is in the slip condition and remains in motion until $\dot{x}_{i}=V_{i}$. Next, if $\dot{x}_{i}=V_{i}$ at a certain moment, the motion just after that moment is determined from the inequality relation between the static friction force keeping the block resting against the belt and the maximum static friction force. Setting the coefficient of static friction to $\mu_{s i}$, the motion just after that moment becomes slip if $\left|f_{s i}\right|>\mu_{s i} g$ or stick if $\left|f_{s i}\right|<\mu_{s i} g$. Therefore, the switching condition is as follows when Block $i$ is in the slip condition:

$$
\dot{x}_{i}-V_{i}=0 \text {. }
$$

When Block $i$ is in the stick condition, it is

$$
\left|f_{s i}\right|-\mu_{s i} g=0 \text {. }
$$

\section{Numerical method for computing synchronized solutions}

\subsection{Application of the shooting method $^{(7)}$}

In the present report, the steady periodic solutions in which stick-slip motion occurs in both oscillators are defined as synchronized solutions, and accurate approximations are computed using the shooting method. To apply the shooting method, the equation of motion is transformed to the following first-order ordinary differential equation in normal form:

$$
\dot{\boldsymbol{y}}=\boldsymbol{Y}(\boldsymbol{y}), \quad \boldsymbol{y}=\left(y_{1}, y_{2}, y_{3}, y_{4}\right)^{T}=\left(x_{1}, x_{2}, \dot{x}_{1}, \dot{x}_{2}\right)^{T}, \quad \boldsymbol{Y}(\boldsymbol{y})=\left(Y_{1}, Y_{2}, Y_{3}, Y_{4}\right)^{T},
$$

where the superscript " $T$ " denotes the transpose operation. It is necessary to switch $\boldsymbol{Y}(\boldsymbol{y})$ according to the block motions. When both blocks are in the slip condition, $\boldsymbol{Y}(\boldsymbol{y})$ is written as follows:

$$
Y_{i}=y_{i+2}, \quad Y_{i+2}=\mu_{d i} g-2 \zeta_{i} \omega_{i} y_{i+2}-\omega_{i}^{2} y_{i}-2 \tilde{\zeta}_{i} \tilde{\omega}_{i}\left(y_{i+2}-y_{j+2}\right)-\tilde{\omega}_{i}^{2}\left(y_{i}-y_{j}\right) .
$$

On the other hand, when Block $i$ is in the stick condition, $\boldsymbol{Y}(\boldsymbol{y})$ is given by

$$
Y_{i}=y_{i+2}=V_{i}, \quad Y_{i+2}=0 .
$$

In addition, in the shooting method, the equation of variation for Eq. (9)

$$
\dot{\eta}=\Psi(y) \eta, \quad \Psi(y)=\frac{\partial \boldsymbol{Y}(\boldsymbol{y})}{\partial \boldsymbol{y}}
$$


is also solved with Eq. (9). The coefficient matrix $\Psi(y)$ is also simultaneously switched with $\boldsymbol{Y}(\boldsymbol{y})$.

Setting the initial value of the synchronized solution and the period to $\boldsymbol{y}^{0}$ and $T$, respectively, they satisfy the following equation:

$$
\int_{0}^{T} \boldsymbol{Y}\left\{\boldsymbol{y}\left(t, \boldsymbol{y}^{0}\right)\right\} d t=\mathbf{0},
$$

where $\boldsymbol{y}\left(t, \boldsymbol{y}^{0}\right)$ is the general solution of Eq. (9) with an initial value $\boldsymbol{y}^{0}$. In the shooting method, $\boldsymbol{y}^{0}$ and $T$ are obtained by solving Eq. (13). However, it is necessary to add a phase constraint for $\boldsymbol{y}^{0}$ because Eq. (13) lacks a conditional equation.

$$
g\left(\boldsymbol{y}^{0}\right)=0
$$

Equations (13) and (14) are successively approximated using the Newton method. Setting the initial approximate values obtained by the $k$ th iteration step to $\boldsymbol{y}_{k}^{0}$ and $T_{k}$, those correction values to $\Delta \boldsymbol{y}_{k}^{0}$ and $\Delta T_{k}$, and $\boldsymbol{y}_{k}^{1}=\boldsymbol{y}\left(T_{k}, \boldsymbol{y}_{k}^{0}\right)$, the process of the successive approximation is derived as follows:

$$
\begin{aligned}
& \left.\left[\begin{array}{cc}
\boldsymbol{\Phi}\left(T_{k}\right)-\boldsymbol{I} & \boldsymbol{Y}\left(\boldsymbol{y}_{k}^{1}\right) \\
\partial g\left(\boldsymbol{y}_{k}^{0}\right) / \partial \boldsymbol{y}^{0} & 0
\end{array}\right]\left[\begin{array}{c}
\Delta \boldsymbol{y}_{k}^{0} \\
\Delta T_{k}
\end{array}\right]=\left[\begin{array}{c}
\boldsymbol{y}_{k}^{0}-\boldsymbol{y}_{k}^{1} \\
-g\left(\boldsymbol{y}_{k}^{0}\right)
\end{array}\right],\left[\begin{array}{l}
\boldsymbol{y}_{k+1}^{0} \\
T_{k+1}
\end{array}\right]=\left[\begin{array}{c}
\boldsymbol{y}_{k}^{0}+\Delta \boldsymbol{y}_{k}^{0} \\
T_{k}+\Delta T_{k}
\end{array}\right]\right\}, \\
& (k=0,1,2, \cdots)
\end{aligned}
$$

where $\Phi(t)$ is the state transition matrix (the fundamental solution matrix with an initial value of the identity matrix $\boldsymbol{I}$ ) of Eq. (12). The values of $\boldsymbol{y}_{k}^{T}$ and $\boldsymbol{\Phi}\left(T_{k}\right)$ in Eq. (15) can be computed by numerically integrating Eqs. (9) and (12) from $t=0$ to $t=T_{k}$. However, to analyze the stick-slip motion, it is necessary to introduce a special procedure to calculate the variation $\eta$ before and after the switching time; this procedure is shown in $\S 3.2$.

\subsection{Calculation procedure for discontinuities of variation}

The switching conditions in Eqs. (7) and (8) are given as a function of $\boldsymbol{y}$ and $\boldsymbol{Y}$ and are collectively expressed as follows:

$$
q(\boldsymbol{y}, \boldsymbol{Y})=0 .
$$

In the present model, the value of $\boldsymbol{Y}$ varies discontinuously at the switching time. In addition, the discontinuity generated in $\eta$ can be expressed as Eq. (17) using the procedure introduced in the previous report ${ }^{(8)}$,

$$
\eta^{+}=\boldsymbol{B} \eta^{-}, \quad \boldsymbol{B}=\left[\boldsymbol{I}+\frac{\Delta \boldsymbol{Y} \hat{\boldsymbol{Q}}}{\hat{\boldsymbol{Q}} \hat{\boldsymbol{Y}}}\right], \quad \hat{\boldsymbol{Q}}=\frac{\partial q(\hat{\boldsymbol{y}}, \hat{\boldsymbol{Y}})}{\partial \boldsymbol{y}}+\frac{\partial q(\hat{\boldsymbol{y}}, \hat{\boldsymbol{Y}})}{\partial \boldsymbol{Y}} \boldsymbol{\Psi}(\hat{\boldsymbol{y}})
$$

where the superscripts " $-"$ and $"+"$ denote the variations just before and after the switching time, respectively, and the head mark " $\wedge$ " indicates the state variables just before the switching time. In addition, $\Delta \boldsymbol{Y}$ indicates the discontinuity of $\boldsymbol{Y}$ at the switching time (i.e., the difference between the values of $\boldsymbol{Y}$ just after and before the switching time) and is obtained as follows when the motion switches:

$$
\Delta \boldsymbol{Y}=\left(0,0, \Delta Y_{3}, 0\right)^{T} \quad(i=1), \quad \Delta \boldsymbol{Y}=\left(0,0,0, \Delta Y_{4}\right)^{T} \quad(i=2) .
$$

If Block $i$ is in the slip condition just before the switching time, $\hat{Q}$ is written as follows using Eq. (7):

$$
\hat{\boldsymbol{Q}}=(0,0,1,0) \quad(i=1), \quad \hat{\boldsymbol{Q}}=(0,0,0,1) \quad(i=2) .
$$

Meanwhile, if Block $i$ is in the stick condition, $\hat{Q}$ is obtained as follows using Eq. (8):

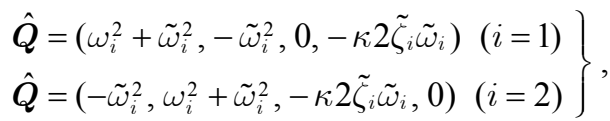

where $\kappa$ is one when the motion of Block $j$ is slip or zero when it is stick.

As indicated above, in order to analyze the stick-slip motion using the shooting method, 


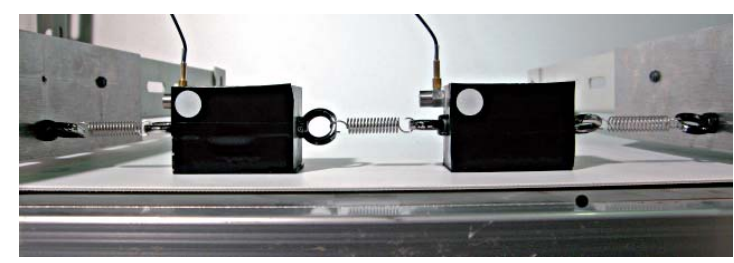

Fig.2 Photograph of experimental apparatus

Table 1 System parameters

\begin{tabular}{c|c||c|c||c|c}
\hline \hline$m_{1}[\mathrm{~kg}]$ & 0.137 & $c_{2}[\mathrm{~N} \cdot \mathrm{s} / \mathrm{m}]$ & 0.030 & $\mu_{s 2}[-]$ & 0.82 \\
$m_{2}[\mathrm{~kg}]$ & 0.137 & $C[\mathrm{~N} \cdot \mathrm{s} / \mathrm{m}]$ & 0.025 & $V_{1}[\mathrm{~m} / \mathrm{s}]$ & 0.20 \\
$k_{1}[\mathrm{~N} / \mathrm{m}]$ & 212.0 & $\mu_{d 1}[-]$ & 0.43 & $V_{2}[\mathrm{~m} / \mathrm{s}]$ & 0.20 \\
$k_{2}[\mathrm{~N} / \mathrm{m}]$ & 212.5 & $\mu_{d 2}[-]$ & 0.43 & & \\
$c_{1}[\mathrm{~N} \cdot \mathrm{s} / \mathrm{m}]$ & 0.030 & $\mu_{s 1}[-]$ & 0.82 & & \\
\hline
\end{tabular}

it is necessary to accurately calculate the switching time and the values of $\hat{y}, \hat{\boldsymbol{Y}}, \Delta \boldsymbol{Y}, \hat{\boldsymbol{Q}}$, and $\eta^{-}$just before the switching time and then to calculate the value of $\eta^{+}$just after the switching time using Eq. (17).

\subsection{Stability analysis}

The synchronized solution is computed using the above numerical procedure. Then, the stability can be determined from the characteristic multipliers as the eigenvalues of the state transition matrix $\Phi(T)$. That is, since the present model is an autonomous system, one of them should be exactly one. If all of the absolute values of the other characteristic multipliers are less than one, the synchronized solution is asymptotically stable; otherwise, it is generally unstable.

\section{Experimental setup and method}

The experimental setup produced to validate the present model is outlined in Fig. 2. In this setup, coil springs connect the blocks to each other and to the fixed base in series, as shown in Fig. 1. Both blocks are placed on a belt conveyor so that the direction of block movement aligns with the direction of coil spring deformation. The blocks are placed on the same conveyor because of a constraint of the experimental setup; therefore, the belt speeds of both oscillators are the same $\left(V_{1}=V_{2}\right)$. In addition, rubber sheets are attached to the bottom surfaces of the blocks to increase the difference between the coefficient of dynamic friction and the coefficient of static friction.

In the experiments, the conveyor was driven at a constant speed, and synchronization was checked based on the frequency analysis of the acceleration responses from pickups installed on each of the blocks. When synchronization occurred, the positions of the observation points (the white circles shown in Fig. 2) on each of the blocks were measured at 200 frames per second for three seconds using a motion capture system (Real Time Tracker Radish from Library).

Preliminary examinations confirmed that synchronization occurs only when the individual difference between the oscillators is small and occurs over a wide range of spring constant $K$ of the coil spring coupling the blocks when the difference is small. Therefore, in the present report, the spring constant $K$ was varied using several coil springs with a small individual difference, and the present model was validated by comparing the numerical and experimental results.

\section{Numerical and experimental results}

\subsection{Numerical conditions}

Table 1 shows the system parameters obtained from the experimental setup shown in 
Fig. 2, with the exception of the spring constant $K$. These values were obtained by averaging the actual measurements of both oscillators. The values of the spring constants $k_{1}$ and $k_{2}$, however, were set as described below. At first, each block was connected individually to the fixed wall by a coil spring whose spring constant according to the catalog was identical, and then we experimentally observed the stick-slip motion generated in each of the blocks. As a result, a slight difference was confirmed in the frequencies of both oscillators. Therefore, in order to express the individual difference between the oscillators, the values of $k_{1}$ and $k_{2}$ were slightly changed from the value according to the catalog.

In the calculation, the synchronized solutions were obtained using the numerical method described in $\S 3$. The Runge-Kutta-Gill method was used for numerical integration in the shooting method; the number of divisions per period was set to 2048, and the results were computed using double precision. In the successive approximation procedure for calculating the synchronized solutions and the switching time, the convergence criterion for the relative error was set to $10^{-12}$ or less. Meanwhile, the convergence process of the synchronized solution showed close secondary convergence using the theoretical Newton method. In addition, one of the characteristic multipliers that should be exactly one was obtained accurately. Therefore, these results validated the effectiveness of the procedure described in $\$ 3.2$ at dealing with the discontinuity of the variation. All of the synchronized solutions obtained in this report were confirmed to be very accurate.

\subsection{Validation of analytical model}

Figure 3 shows the experimental and numerical results obtained by changing the spring constant $K$. The abscissa shows the spring constant $K$, and the ordinates show the synchronized frequency $f_{\text {syn }}$, the double amplitude values $\operatorname{Amp}\left(x_{1}\right)$ and $\operatorname{Amp}\left(x_{2}\right)$, and the stick ratio, which is defined as the ratio of the time during which Block $i$ is in the stick condition to the period. However, the stick ratio was difficult to obtain in the experiment, so only the numerical results are shown. In this figure, the @ symbols denote the experimental results, the solid black lines indicate the stable solutions (SS), and the dashed lines indicate the unstable solutions (US) obtained from the computations. On the solution branches, the O symbols denote the saddle-node bifurcation points (SN), the $\nabla$ symbols denote the period-doubling bifurcation points (PD), and the $\square$ symbols indicate the limit at which the synchronized solutions occurred (the reason for generating this limit is discussed in §6). In the experimental results, all of the data obtained with the coil springs that were available for this experiment are shown. In addition, the red lines in the graph of the synchronized frequency $f_{\text {syn }}$ denote the first natural frequency $f_{n 1}$ and the second natural frequency $f_{n 2}$ obtained from the linear two-degree-of-freedom system in which the Coulomb friction and the viscous damping forces are removed.

As seen in Fig. 3, two types of synchronized solutions exist along the first and second natural frequencies $f_{n 1}$ and $f_{n 2}$ of the linear two-degree-of-freedom system. They are denoted as Mode 1 and Mode 2 in order of increasing synchronized frequency $f_{\text {syn }}$. Comparing the experimental and numerical results in the region of $K<400 \mathrm{~N} / \mathrm{m}$, which contains a relatively large amount of experimental data, a slight difference is observed in the existence region of the stable synchronized solutions. However, considering that the stick-slip motion caused by Coulomb friction is easily affected by the surrounding environment and has difficulty maintaining reproducibility, both results agree well. In particular, in the computations, the unstable regions existing locally on the branch of Mode 1 are distinctive. In the experiments performed in the unstable regions using spring constants $K$ of 17.8, 32.0, 174.0, and $212.0 \mathrm{~N} / \mathrm{m}$, although various initial conditions were provided, only Mode 2 occurred as the synchronized solutions. On the other hand, in the region of $K>400 \mathrm{~N} / \mathrm{m}$, we only performed the experiments using spring constants $K$ of 


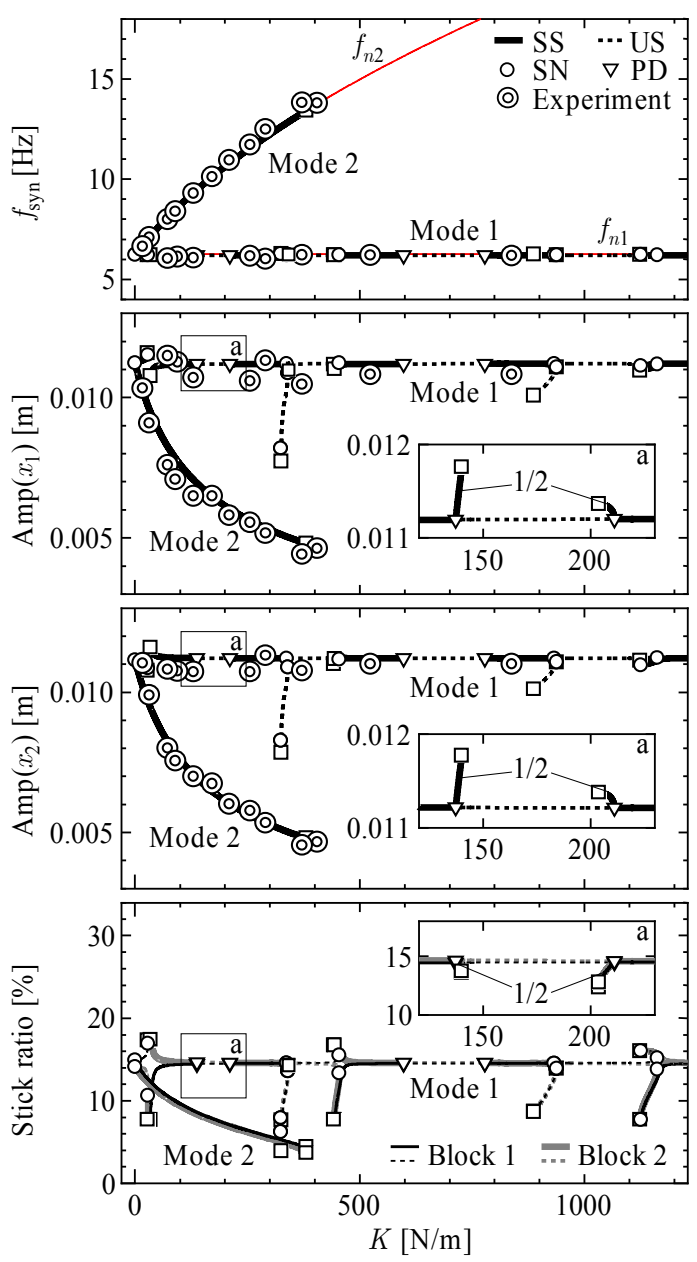

Fig.3 Numerical and experimental results

$528.0,845.0 \mathrm{~N} / \mathrm{m}$ (in which Mode 1 is stable in the computation), and confirmed that Mode 1 was generated in the experiments. These results demonstrate the validity of the present model. Therefore, in what follows, the characteristics and mechanism are investigated mainly based on the numerical results.

\subsection{Types and characteristics of synchronized solutions}

On the solution branches, Mode 1 and Mode 2, stable synchronized solutions with different vibratory patterns exist. Figure 4 shows example time histories of the stable synchronized solutions for $K=132.0 \mathrm{~N} / \mathrm{m}$. The black and red lines represent displacement waveforms of Block 1 and Block 2, respectively. The numerical results agree well with the experimental results in both Mode 1 and Mode 2. The characteristics of the synchronized solutions are described below.

First, as shown in Fig. 4(a), both blocks vibrate nearly in phase in Mode 1, and the vibratory pattern is close to the first natural mode. The synchronized frequency $f_{\mathrm{syn}}$ is slightly lower than the first natural frequency $f_{n 1}$ and is nearly constant against the spring constant $K$. Because the blocks vibrate nearly in phase, the amplitudes are almost the same; hence, the relative displacement is very small, and the change in $K$ does not significantly affect the synchronized frequency. On the other hand, the magnitude of $K$ affects the stability of Mode 1 . The unstable regions based on the saddle-node and period-doubling bifurcation points alternate on the Mode 1 branch (the reason it generates an unstable region is examined in §7). The synchronized frequency and the stick ratio complexly change in the neighborhood of the saddle-node bifurcation points. In addition, as 

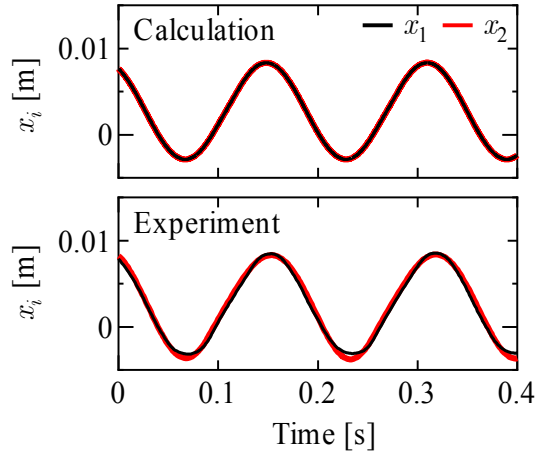

(a) Mode 1
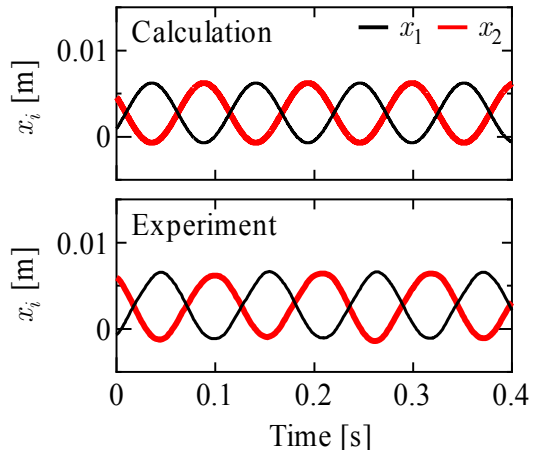

(b) Mode 2

Fig.4 Time histories of stable synchronized solution $(K=132.0 \mathrm{~N} / \mathrm{m})$

shown in the enlarged figure [Fig. 3a], the stable synchronized solution with the double period (the fundamental frequency is half of $f_{\text {syn }}$ ) is generated in the very narrow regions from the period-doubling bifurcation points.

Next, as shown in Fig. 4(b), the blocks vibrate nearly out of phase in Mode 2, and the vibratory pattern is close to the second natural mode. The synchronized frequency $f_{\text {syn }}$ is slightly lower than the second natural frequency $f_{n 2}$. Both $f_{\text {syn }}$ and $f_{n 2}$ increase with the spring constant $K$, but the amplitudes and the stick ratio of both blocks decrease. Mode 2 does not exist for $K>381.5 \mathrm{~N} / \mathrm{m}$ because the energy adjustment mechanism for realizing the synchronization does not work there due to the decreasing stick ratio.

As described above, the synchronization generated in the present model correlates well with the undamped free vibration characteristics of the linear two-degree-of-freedom system without Coulomb friction. In addition, compared with the results obtained in the previous reports ${ }^{(4)-(6)}$, there are only two types of synchronized solutions, and the solution structure is relatively simple, with the exception of the regions close to the saddle-node bifurcation point. From these results, the condition for generating synchronization and the mechanism are examined in $\S 6$ based on the energy transmission between the oscillators.

\section{Occurrence condition and mechanism of synchronized solutions}

\subsection{Examination targets and evaluated quantities}

In the present model, energy transmission between the oscillators through the coupling element should realize synchronization by varying the switching times, widths and positions of the stick regions, amplitudes, phase angles, and so on. In this section, to clarify the adjustment mechanism, we assume a system in which the viscous damping coefficients and the coefficients of dynamic friction are set to zero $\left(c_{1}=c_{2}=C=0.0 \mathrm{~N} \cdot \mathrm{s} / \mathrm{m}\right.$ and $\mu_{d 1}=$ $\mu_{d 2}=0.0$ ), and the coefficients of static friction are set to the difference between the former coefficients of dynamic and static friction. Systems having the following two types of individual differences between the oscillators are investigated:

(i) $\mu_{s 1} \neq \mu_{s 2}$ : the case in which the nonlinearities of the oscillators differ;

(ii) $k_{1} \neq k_{2}$ : the case in which the free vibration characteristics of the oscillators differ.

The other system parameters are the same as in Table 1, with the exception of the above ones, and $K=100.0 \mathrm{~N} / \mathrm{m}$. Setting the coefficients of dynamic and static friction as described above is equivalent to rewriting the equation of motion and the switching condition using the relative displacements from the static equilibrium point when both blocks are always in the slip condition. The types and characteristics of the synchronized solutions generated in the models used in this section are the same as in $\S 5$.

In such systems, the mechanical energy of the system is conserved when both blocks are in the slip condition; on the other hand, the friction forces feed or dissipate energy when either block is in the stick condition. In these cases, a change in block motion occurs four 
times per period $T$. Setting the time at which the motion of Block 1 switches from stick to slip to $t_{0}$ and setting the later switching times to $t_{1}, t_{2}, t_{3}$, and $t_{4}$ (i.e., $t_{4}=t_{0}+T$ ) in that order, the time interval from $t_{p-1}$ to $t_{p},(p=1,2,3,4)$ is denoted as the interval $p$. In addition, the variation in the mechanical energy of Block $i \quad \Delta E_{i, p}$ and that of the work done by the restoring force $-K\left(x_{i}-x_{j}\right)$ (hereinafter referred to as the coupling force) $\Delta W_{i, p}$ during the interval $p$ are respectively defined as follows:

$$
\Delta E_{i, p}=\int_{t_{p-1}}^{t_{p}}\left\{m_{i} \ddot{x}_{i}+k_{i} x_{i}+K\left(x_{i}-x_{i}\right)\right\} \dot{x}_{i} d \tau, \Delta W_{i, p}=-\int_{t_{p-1}}^{t_{p}} K\left(x_{i}-x_{i}\right) \dot{x}_{i} d \tau .
$$

Furthermore, the total variation in the mechanical energy of Block $i$ and that of the work of the coupling force acting on Block $i$ from $t_{0}$ to $t_{q}(q=1,2,3,4)$ are given by

$$
E_{i, q}=\sum_{p=1}^{q} \Delta E_{i, p}, \quad W_{i, q}=\sum_{p=1}^{q} \Delta W_{i, p} .
$$

In the following subsections, we regard the physical quantities shown in Eqs. (21) and (22) as the evaluated quantities for measuring the influence of the interaction between the oscillators and then examine the amount of energy transition between the oscillators when the synchronization is generated. In the two systems studied in this section, when synchronization is generated, the variation in the total mechanical energy during the period becomes zero because the viscous damping forces do not act; that is,

$$
E_{1,4}+E_{2,4}=0 \text {. }
$$

\subsection{Case of $\mu_{s 1} \neq \mu_{s 2}$}

The coefficients of static friction for Block 1 and Block 2 were set to $\mu_{s 1}=0.10$ and $\mu_{s 2}=0.39$, respectively, and calculations were then performed for $k_{1}=k_{2}=212.0 \mathrm{~N} / \mathrm{m}$. As a result, the stable synchronized solutions corresponding to Mode 1 and Mode 2 studied in the previous section were obtained. Figure 5 shows diagram and velocity waveform of the sable synchronized solutions. The black lines indicate the synchronized solution of Block 1 , and the red lines indicate that of Block 2. Both blocks vibrate nearly in phase each other in Mode 1 and nearly out of phase each other in Mode 2, and the trajectory of Block 1 and that of Block 2 on the phase diagram are axisymmetric one another with respect to $x=0$ (i.e., the amplitudes of the blocks are the same).

Tables 2 and 3 show the values of $\Delta E_{i, p}, \Delta W_{i, p}, E_{i, q}$, and $W_{i, q}$ and the block motions of Mode 1 and Mode 2 in the interval $p$. In both solutions, for Block 1 , the stick region of $x<0$ is wide, and $\Delta E_{1,1}<0$ and $\Delta E_{1,2}<0$ in Mode $1 ; \Delta E_{1,1}<0$ in Mode 2. By contrast, for Block 2, the stick region of $x>0$ is wide, and $\Delta E_{2,2}>0$ and $\Delta E_{2,3}>0$ in Mode 1; $\Delta E_{2,3}>0$ in Mode 2. This is due to the fact that energy is dissipated since the static friction force does almost negative work on Block $i$ in the stick region of $x_{i}<0$, and energy is supplied since the static friction force does almost positive work on Block $i$ in the region of $x_{i}>0$. In both Mode 1 and Mode 2, with regard to the variations in mechanical energy and work done by the coupling force during a period, $E_{1,4}<0, W_{1,4}>0$ and $E_{1,4}+W_{1,4}=0$ for Block 1, $E_{2,4}>0, W_{2,4}<0$ and $E_{2,4}+W_{2,4}=0$ for Block 2, and $E_{1,4}+E_{2,4}=0$ and $W_{1,4}+W_{2,4}=0$ for the whole system. This indicates
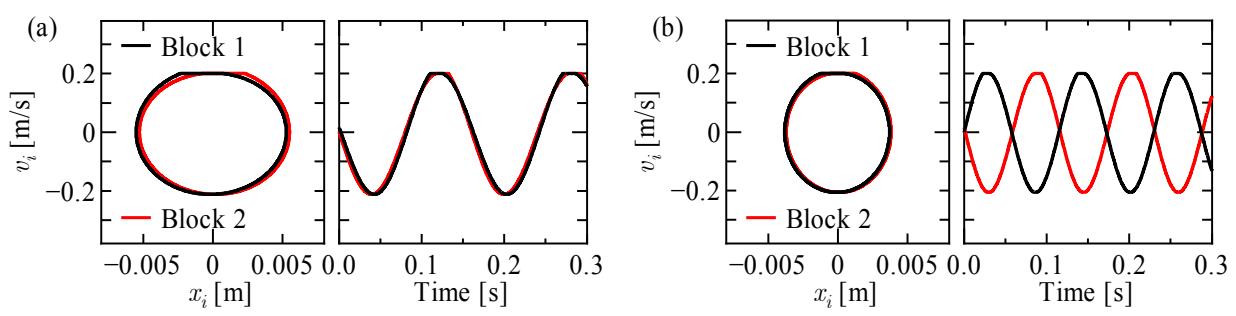

Fig.5 Phase diagram of synchronized solutions: (a) Mode 1 and (b) Mode 2 $\left(k_{1}=k_{2}=212.0 \mathrm{~N} / \mathrm{m}, K=100.0 \mathrm{~N} / \mathrm{m}, \mu_{\mathrm{s} 1}=0.10, \mu_{\mathrm{s} 2}=0.39\right)$ 
Table 2 Energy transition of Mode 1

\begin{tabular}{c|r|r|c|c}
$\left(k_{1}=k_{2}=212.0 \mathrm{~N} / \mathrm{m}, K=100.0 \mathrm{~N} / \mathrm{m}, \mu_{\mathrm{s} 1}=0.10, \mu_{\mathrm{s} 2}=0.39\right)$ \\
\hline \hline$p$ & 1 & 2 & 3 & 4 \\
\hline \hline$E_{1, p}[\mathrm{~J}]$ & $\times 10^{-5}$ & $\times 10^{-5}$ & $\times 10^{-5}$ & $\times 10^{-5}$ \\
$\Delta E_{2, p}[\mathrm{~J}]$ & 0.0 & 9.84 & 49.9 & 0.0 \\
$E_{1, p}[\mathrm{~J}]$ & -49.9 & -59.8 & -59.8 & -59.8 \\
$E_{2, p}[\mathrm{~J}]$ & 0.0 & 9.84 & 59.8 & 59.8 \\
$\Delta W_{1, p}[\mathrm{~J}]$ & 3.62 & 4.78 & 3.52 & 47.9 \\
$\Delta W_{2, p}[\mathrm{~J}]$ & -3.52 & -4.78 & -3.62 & -47.9 \\
$W_{1, p}[\mathrm{~J}]$ & 3.62 & 8.39 & 11.9 & 59.8 \\
$W_{2, p}[\mathrm{~J}]$ & -3.52 & -8.30 & -11.9 & -59.8 \\
\hline \hline Block 1 & stick & stick & slip & slip \\
Block 2 & slip & stick & stick & slip
\end{tabular}

Table 3 Energy transition of Mode 2

\begin{tabular}{c|r|r|r|r}
\hline \hline \multicolumn{1}{c|}{$p$} & \multicolumn{1}{c|}{1} & \multicolumn{1}{c|}{2} & \multicolumn{1}{c}{3} & \multicolumn{1}{c}{4} \\
\hline \hline & $\times 10^{-5}$ & $\times 10^{-5}$ & $\times 10^{-5}$ & $\times 10^{-5}$ \\
$\Delta E_{1, p}[\mathrm{~J}]$ & -31.2 & 0.0 & 0.0 & 0.0 \\
$\Delta E_{2, p}[\mathrm{~J}]$ & 0.0 & 0.0 & 31.2 & 0.0 \\
$E_{1, p}[\mathrm{~J}]$ & -31.2 & -31.2 & -31.2 & -31.2 \\
$E_{2, p}[\mathrm{~J}]$ & 0.0 & 0.0 & 31.2 & 31.2 \\
$\Delta W_{1, p}[\mathrm{~J}]$ & 17.5 & 14.2 & -17.3 & 16.8 \\
$\Delta W_{2, p}[\mathrm{~J}]$ & 1.62 & 1.45 & -17.5 & -16.8 \\
$W_{1, p}[\mathrm{~J}]$ & 17.5 & 31.7 & 14.4 & 31.2 \\
$W_{2, p}[\mathrm{~J}]$ & 1.62 & 3.07 & -14.4 & -31.2 \\
\hline \hline Block 1 & stick & slip & slip & slip \\
Block 2 & slip & slip & stick & slip \\
\hline
\end{tabular}

that the energy supplied in the stick region of Block 2 transfers to Block 1 through the coupling element, and then all the energy dissipates in the stick region of Block 1. Besides, for $\mu_{s 1}>\mu_{s 2}$, the results can be obtained by exchanging Block 1 for Block 2 (these details are omitted). Therefore, synchronization is realized for $\mu_{s 1} \neq \mu_{s 2}$ by transmitting the energy from the block having the higher coefficient of static friction to the block having the lower one.

Based on these results, we discuss the reason why the energy transition realizes synchronization for $\mu_{s 1} \neq \mu_{s 2}$. First, considering the case in which the coupling element does not exist and each block independently has stick-slip motion, the stick region of Block 2 is wide and the period is long for $\mu_{s 1}<\mu_{s 2}$. The mechanical energy of Block 2 in the slip region is also high since the amplitude of Block 2 is large. In addition, because the viscous damping forces and dynamic friction forces do not act on the blocks, the trajectories of the blocks on the phase plane are axisymmetric with respect to $x=0$, and the work done by the static friction force in the entire stick region becomes zero. In order to realize synchronization when such oscillators are coupled, it is necessary to make the width of the 
stick regions and the amplitudes of both blocks agree because the other parameters are the same, with the exception of the coefficients of static friction. This is the reason why energy transfers from Block 2 to Block 1 through the coupling element in both solutions. For realizing such energy transition, the stick region of $x>0$ for Block 2 should be wide, and the trajectories of Block 1 and Block 2 should be axisymmetric with respect to $x=0$. Besides, setting the displacement at the moment when the motion of Block 1 switches from stick to slip to $\hat{X}(>0)$ (i.e., the displacement at which the motion of Block 2 switches from slip to stick is $-\hat{X}$ ), $\hat{X}$ asymptotically moves closer to zero (the static equilibrium point) as $\mu_{s 1}$ decreases, and the value of $\hat{X}$ becomes a small positive real number at the limit at which synchronization occurs. In addition, for $\mu_{s 1}>\mu_{s 2}$, Block 1 should be exchanged with Block 2 in the above investigation.

\subsection{Case of $k_{1} \neq k_{2}$}

The spring constants $k_{1}$ and $k_{2}$ were set to $200.0 \mathrm{~N} / \mathrm{m}$ and $212.0 \mathrm{~N} / \mathrm{m}$, and the calculations were then performed for $\mu_{s 1}=\mu_{s 2}=0.39$. As a result, the stable synchronized solutions corresponding to Mode 1 and Mode 2 studied in the previous section were obtained. Figure 6 shows the synchronized solutions on the phase diagram and the velocity waveforms. The black lines show the synchronized solution of Block 1, and the red lines show that of Block 2. Both blocks vibrate nearly in phase each other in Mode 1 and nearly out of phase each other in Mode 2. The amplitude and width of the stick region of Block 1 are larger than those of Block 2 in Mode 1; in contrast, those of Block 2 are larger than those of Block 1 in Mode 2.

Tables 4 and 5 show the same parameters as Tables 2 and 3. In Mode 1, the stick region of $x<0$ for Block 1 is wide, $E_{1,4}<0, W_{1,4}>0$, and $E_{1,4}+W_{1,4}=0$; on the other hand, the stick region of $x>0$ for Block 2 is wide, $E_{2,4}>0, W_{2,4}<0$, and $E_{2,4}+W_{2,4}=0$. Besides, $E_{1,4}+E_{2,4}=0$ and $W_{1,4}+W_{2,4}=0$ for the whole system, which shows that the energy supplied in the stick region of Block 2 transfers to Block 1 through the coupling element, and all of the energy dissipates in the stick region of Block 1. In addition, in Mode 2, the characteristics of Block 1 and Block 2 are opposite to those in Mode 1; the energy transfers from Block 1 to Block 2 when synchronization occurs. Besides, the results can be obtained by exchanging Block 1 for Block 2 in the above investigation for $k_{1}>k_{2}$ (these details are omitted). As just described, for $k_{1} \neq k_{2}$, synchronization is realized by transmitting the energy from the block having the smaller amplitude to the block having the larger amplitude.

Based on these results, we discuss the reason why the energy transition realizes Mode 1 [Table 4 and Fig. 6(a)] for $k_{1} \neq k_{2}$. For this purpose, we develop a virtual system that has a synchronized solution of the same frequency as Mode 1 described above for $k_{1}=k_{2}$; this system is called the standard system. In the standard system, the relative displacement between oscillators is always zero in Mode 1 since the parameters of both oscillators are the same. The spring constants obtained from the synchronized frequency of Mode 1 shown in Table 4 are set to $k_{1}=k_{2}=205.39 \mathrm{~N} / \mathrm{m}$ (the other parameters are the same as those in
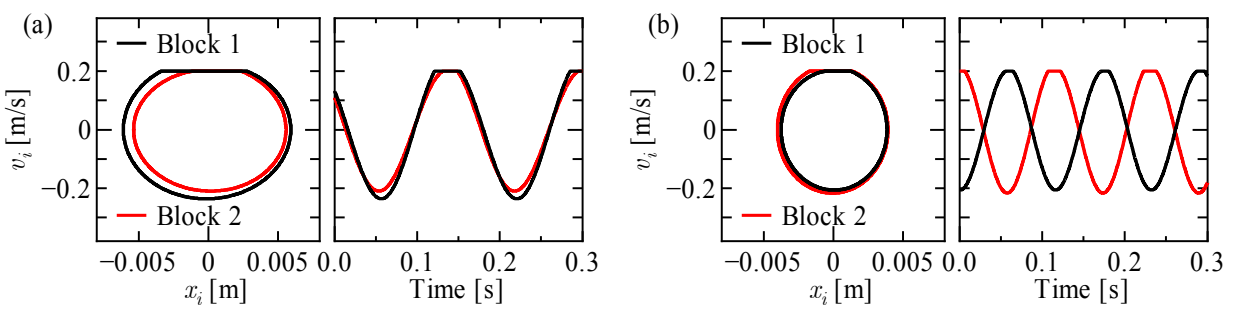

Fig.6 Phase diagram of synchronized solutions: (a) Mode 1 and (b) Mode 2 $\left(k_{1}=200.0 \mathrm{~N} / \mathrm{m}, k_{2}=212.0 \mathrm{~N} / \mathrm{m}, K=100.0 \mathrm{~N} / \mathrm{m}, \mu_{\mathrm{s} 1}=\mu_{\mathrm{s} 2}=0.39\right)$ 
Table 4 Energy transition of Mode 1

\begin{tabular}{c|r|r|r|r}
$\left(k_{1}=200.0 \mathrm{~N} / \mathrm{m}, k_{2}=212.0 \mathrm{~N} / \mathrm{m}, K=100.0 \mathrm{~N} / \mathrm{m}, \mu_{\mathrm{s} 1}=\mu_{\mathrm{s} 2}=0.39\right)$ \\
\hline \hline$p$ & 1 & 2 & 3 & 4 \\
\hline \hline & $\times 10^{-5}$ & $\times 10^{-5}$ & $\times 10^{-5}$ & $\times 10^{-5}$ \\
$\Delta E_{1, p}[\mathrm{~J}]$ & -101 & 17.9 & 28.9 & 0.0 \\
$\Delta E_{2, p}[\mathrm{~J}]$ & 0.0 & 54.9 & 0.0 & 0.0 \\
$E_{1, p}[\mathrm{~J}]$ & -101 & -83.8 & -54.9 & -54.9 \\
$E_{2, p}[\mathrm{~J}]$ & 0.0 & 54.9 & 54.9 & 54.9 \\
$\Delta W_{1, p}[\mathrm{~J}]$ & 5.90 & 8.58 & 1.53 & 38.9 \\
$\Delta W_{2, p}[\mathrm{~J}]$ & -5.49 & -8.58 & -1.49 & -39.3 \\
$W_{1, p}[\mathrm{~J}]$ & 5.90 & 14.5 & 16.0 & 54.9 \\
$W_{2, p}[\mathrm{~J}]$ & -5.49 & -14.1 & -15.6 & -54.9 \\
\hline \hline Block 1 & stick & stick & stick & slip \\
Block 2 & slip & stick & slip & slip
\end{tabular}

Table 5 Energy transition of Mode 2

$\left(k_{1}=200.0 \mathrm{~N} / \mathrm{m}, k_{2}=212.0 \mathrm{~N} / \mathrm{m}, K=100.0 \mathrm{~N} / \mathrm{m}, \mu_{\mathrm{s} 1}=\mu_{\mathrm{s} 2}=0.39\right)$

\begin{tabular}{c|r|r|r|r}
\hline \hline$p$ & \multicolumn{1}{c|}{1} & \multicolumn{1}{c|}{2} & \multicolumn{1}{c|}{3} & \multicolumn{1}{c}{4} \\
\hline \hline & $\times 10^{-5}$ & $\times 10^{-5}$ & $\times 10^{-5}$ & $\times 10^{-5}$ \\
$\Delta E_{1, p}[\mathrm{~J}]$ & 30.0 & 0.0 & 0.0 & 0.0 \\
$E_{2, p}[\mathrm{~J}]$ & 0.0 & 0.0 & -30.0 & 0.0 \\
$E_{1, p}[\mathrm{~J}]$ & 30.0 & 30.0 & 30.0 & 30.0 \\
$E_{2, p}[\mathrm{~J}]$ & 0.0 & 0.0 & -30.0 & -30.0 \\
$\Delta W_{1, p}[\mathrm{~J}]$ & -17.4 & -30.5 & 17.0 & 0.99 \\
$\Delta W_{2, p}[\mathrm{~J}]$ & -18.1 & 4.46 & 18.3 & 25.3 \\
$W_{1, p}[\mathrm{~J}]$ & -17.4 & -48.0 & -31.0 & -30.0 \\
$W_{2, p}[\mathrm{~J}]$ & -18.1 & -13.6 & 4.67 & 30.0 \\
\hline \hline Block 1 & stick & slip & slip & slip \\
Block 2 & slip & slip & stick & slip \\
\hline
\end{tabular}

Table 4, with the exception of $k_{1}$ and $k_{2}$ ). The width of the stick region $X_{s t}$ and the time during which the blocks are in the stick condition $T_{s t}$ are $X_{s t}=0.510 \times 10^{-2} \mathrm{~m}$ and $T_{s t}=0.0255 \mathrm{~s}$, and the width of the slip region $X_{s l}$ (i.e., the double amplitude value) and the time during which the blocks are in the slip condition $T_{s l}$ are $X_{s l}=0.0115 \mathrm{~m}$ and $T_{s l}=0.1386 \mathrm{~s}$, respectively. The same physical quantities of the synchronized solution shown in Table 4 (the superscripts distinguish both blocks) are $X_{s t}^{1}=0.610 \times 10^{-2} \mathrm{~m}$, $T_{s t}^{1}=0.0305 \mathrm{~s}, X_{s l}^{1}=0.0120 \mathrm{~m}$, and $T_{s l}^{1}=0.1336 \mathrm{~s}$ for Block 1 ; in contrast, $X_{s t}^{2}=$ $0.343 \times 10^{-2} \mathrm{~m}, T_{s t}^{2}=0.0172 \mathrm{~s}, X_{s l}^{2}=0.0109 \mathrm{~m}$, and $T_{s l}^{2}=0.1469 \mathrm{~s}$ for Block 2. Based on the fact that the amplitude of Block 1 is larger than that of Block 2 in the first natural mode, $X_{s t}^{1}>X_{s t}, T_{s t}^{1}>T_{s t}, X_{s l}^{1}>X_{s l}$, and $T_{s l}^{1}<T_{s l}$ for Block 1, and $X_{s t}^{2}<X_{s t}, T_{s t}^{2}<T_{s t}, X_{s l}^{2}<$ $X_{s l}$, and $T_{s l}^{2}>T_{s l}$ for Block 2. Therefore, it is proved that synchronization is realized by accelerating Block 1 (having the larger amplitude) to shorten the time during which it is in the slip condition in the standard system and by decelerating Block 2 (having the smaller amplitude) to lengthen the time during which it is in the slip condition. This is the reason why the energy transfers from Block 2 to Block 1 when the blocks are in the slip condition, 
as shown in Table 4. The same investigation can be applied to Mode 2 (these details are omitted). These facts cause the energy transmission from the block with the smaller amplitude to the block with the larger amplitude through the coupling element in both solutions. For realizing such energy transmission, the stick region of $x<0$ for the block with the larger amplitude should be wide, and that of $x>0$ for the block with the smaller amplitude should also be wide. Besides, by setting the displacement at which the motion of the block with the smaller amplitude switches from slip to stick to $-\hat{X}(<0), \hat{X}$ asymptotically moves closer to zero (the static equilibrium point) as $k_{1}$ decreases; the value of $\hat{X}$ becomes a small positive real number for $k_{1}$ at the limit at which synchronization occurs. In addition, for $k_{1}>k_{2}$, Block 1 should be exchanged with Block 2 in the above discussion.

The above is also the reason why the limit at which synchronized solutions occur is generated in Fig. 3. The system investigated in Fig. 3, however, is different in that the static equilibrium point when both blocks are always in the slip condition moves to the positive direction from the origin, and that the difference between the displacement at the switching point (at which the motion switches from slip to stick at the limit of generating synchronized solutions) and the static equilibrium point becomes slightly larger than that of the above case because of the energy dissipation from the viscous damping forces.

\subsection{Summary}

In this section, the mechanism behind synchronization in simplified models was examined from the viewpoint of energy transmission through the coupling element. Suitable energy transmission is the common mechanism behind synchronization generated in various mechanical systems. However, even in such simplified systems, the methods of energy transmission differ depending on the individual difference between the oscillators. This property should make it difficult to integrally clarify the mechanism behind synchronization generated in various vibration systems.

In the present model, in which the oscillators with stick-slip motion are coupled directly, the widths or positions of the stick regions cause the energy adjustment mechanism to realize synchronization. The capability, however, is not high enough and works only when the individual difference is small. Hence, in order to expand the existence region of synchronization, it is necessary to further develop the coupling condition.

\section{Examination of unstable region in Mode 1 branch}

In this section, the reason behind the unstable solution generated in the Mode 1 branch is investigated using the in-phase synchronized solution generated in the system in which two identical undamped oscillators are coupled (denoted as a symmetric system). The in-phase solution in the symmetric system can be obtained analytically since it is the same as the stick-slip motion generated in the system in which either oscillator exists independently. As shown below, the subscript $i$ is removed from the symbols representing the system parameters.

\subsection{In-phase solution generated in symmetric system}

First, we calculate the period of the stick-slip motion $x(t)$. The time origin $(t=0)$ is defined as just after the time at which the motion of the block switches from stick to slip; the initial condition is denoted as $x(0)=\mu_{s} g / \omega_{n}^{2}$ and $\dot{x}(0)=V$. Then, the switching time $\hat{t}$ at which the velocity of the block reaches $V$ again and the displacement of the block at that time $x(\hat{t})$ are given by

$$
\hat{t}=\frac{2\left(\pi-\tan ^{-1} \lambda\right)}{\omega_{n}}, x(\hat{t})=\frac{\mu_{d} g}{\omega_{n}^{2}}-\frac{V \lambda}{\omega_{n}},
$$

where 


$$
\lambda=\frac{\left(\mu_{s}-\mu_{d}\right) g}{\omega_{n} V}, \omega_{n}=\sqrt{\frac{k}{m}} .
$$

The parameter $\lambda$ is a dimensionless number characterizing the stick-slip motion. The bigger the value of $\lambda$, the more easily the stick-slip motion is generated.

The time during which the block is in the stick condition is $\{x(0)-x(\hat{t})\} / V=2 \lambda / \omega_{n}$; therefore, the period $T$ of the stick-slip motion is obtained as follows:

$$
T=\hat{t}+\frac{2 \lambda}{\omega_{n}} .
$$

The switching time and period of the in-phase solution generated in the symmetric system correspond to Eqs. (24) and (26), respectively.

\subsection{State transition matrix}

Here, we obtain the state transition matrix of the equation of variation considering that the motions of both blocks switch at the same instant in a symmetric system.

First, when the blocks are in the slip condition $\left(0^{+} \leq t \leq \hat{t}^{-}\right)$, the equation of variation is given by

$$
\dot{\eta}_{i}=\eta_{i+2}, \quad \dot{\eta}_{i+2}=-\omega_{n}^{2} \eta_{i}-\tilde{\omega}^{2}\left(\eta_{i}-\eta_{j}\right) .
$$

Equation (27) shows a linear two-degree-of-freedom free vibration, and the general solution is easily obtained. Setting the state transition matrix (fundamental solution matrix with an initial value of the identity matrix) of Eq. (27) between $t=0^{+}$and $t=\hat{t}^{-}$to $\Phi\left(\hat{t}^{-}\right)$, the components $\Phi_{p q}(p, q=1, \ldots, 4)$ are obtained as follows:

$$
\left.\begin{array}{l}
\Phi_{11}=\Phi_{22}=\Phi_{33}=\Phi_{44}=\left(C_{1}+C_{2}\right) / 2, \quad \Phi_{21}=\Phi_{12}=\Phi_{43}=\Phi_{34}=\left(C_{1}-C_{2}\right) / 2 \\
\Phi_{31}=\Phi_{42}=-\left(\omega_{n 1} S_{1}+\omega_{n 2} S_{2}\right) / 2, \quad \Phi_{41}=\Phi_{32}=-\left(\omega_{n 1} S_{1}-\omega_{n 2} S_{2}\right) / 2 \\
\Phi_{13}=\Phi_{24}=\left(S_{1} / \omega_{n 1}+S_{2} / \omega_{n 2}\right) / 2, \quad \Phi_{23}=\Phi_{14}=\left(S_{1} / \omega_{n 1}-S_{2} / \omega_{n 2}\right) / 2 \\
C_{1}=\cos \omega_{n 1} \hat{t}, \quad C_{2}=\cos \omega_{n 2} \hat{t}, \quad S_{1}=\sin \omega_{n 1} \hat{t}, \quad S_{2}=\sin \omega_{n 2} \hat{t} \\
\omega_{n 1}=\omega_{n}, \quad \omega_{n 2}=\sqrt{\omega_{n}^{2}+2 \tilde{\omega}^{2}}
\end{array}\right\}
$$

where $\omega_{n 1}$ and $\omega_{n 2}$ are the first and second natural angular frequency of the linear twodegree-of-freedom system without Coulomb friction, respectively. They are also the undamped natural angular frequencies of the equation of variation shown in Eq. (27).

Next, by setting the matrix representing the discontinuity of the variation just before and after $t=\hat{t}$ to $\boldsymbol{B}_{1}$, the value is obtained as follows using Eqs. (10), (11), (17), (18), and (19):

$$
\boldsymbol{B}_{1}=\operatorname{diag}(1,1,0,0) \text {. }
$$

In addition, when the blocks are in the stick condition $\left(\hat{t}^{+} \leq t \leq T^{-}\right)$, the equation of variation is given by $\dot{\eta}_{i}=\dot{\eta}_{i+2}=0$; the state transition matrix does not vary in this interval.

Finally, by setting the matrix representing the discontinuity of the variation just before and after $t=T$ to $\boldsymbol{B}_{2}$, the value is obtained as follows using Eqs. (10), (11), (17), (18), and (20):

$$
\boldsymbol{B}_{2}=\left[\begin{array}{cccc}
1 & 0 & 0 & 0 \\
0 & 1 & 0 & 0 \\
\beta_{1} & \beta_{2} & 1 & 0 \\
\beta_{2} & \beta_{1} & 0 & 1
\end{array}\right], \quad \beta_{1}=-\frac{\left(\omega_{n}^{2}+\tilde{\omega}^{2}\right)\left(\mu_{s}-\mu_{d}\right) g}{\omega_{n}^{2} V}, \quad \beta_{2}=\frac{\tilde{\omega}^{2}\left(\mu_{s}-\mu_{d}\right) g}{\omega_{n}^{2} V} .
$$

Consequently, the transition matrix $\tilde{\Phi}$ from $t=0^{+}$to $t=T^{+}$becomes

$$
\tilde{\boldsymbol{\Phi}}=\boldsymbol{B}_{2} \boldsymbol{B}_{1} \boldsymbol{\Phi}\left(\hat{t}^{-}\right) .
$$




\subsection{Characteristic multiplier}

The ranks of the fourth-order square matrices $\Phi\left(\hat{t}^{-}\right)$and $\boldsymbol{B}_{2}$ are four, and that of $\boldsymbol{B}_{1}$ is two; therefore, the rank of $\tilde{\boldsymbol{\Phi}}$ becomes two. That is, two of the four eigenvalues (characteristic multipliers) of $\tilde{\Phi}$ are exactly zero. In addition, one of them becomes exactly one since the model is an autonomous system. Therefore, the number of nontrivial characteristic multipliers (denoted by $\sigma$ ) is only one, and $\sigma$ is real. The trace of a square matrix is equivalent to the summation of the eigenvalues, which leads to

$$
\sigma=\operatorname{tr} \tilde{\boldsymbol{\Phi}}-1 .
$$

Rearrangement of Eq. (32) along with the substitution of Eqs. (28) (31) yields

$$
\sigma=\frac{\cos \left(\omega_{n 2} \hat{t}+\phi\right)}{\cos \phi}, \cos \phi=\frac{1}{\sqrt{1+\left(\frac{\omega_{n 2}}{\omega_{n 1}} \lambda\right)^{2}}} .
$$

\subsection{Unstable region}

The stability of the in-phase solution in the symmetric system is determined from $\sigma$. That is, saddle-node bifurcation occurs when $\sigma=1$, and period-doubling bifurcation occurs when $\sigma=-1$. The solutions are asymptotically stable when $|\sigma|<1$ and are unstable when $|\sigma|>1$.

From Eqs. (24) and (33), the unstable regions surrounded by the saddle-node bifurcation points are obtained as follows:

$$
\alpha_{r}<\frac{\omega_{n 2}}{\omega_{n 1}}<\frac{r \pi}{\pi-\tan ^{-1} \lambda} .
$$

On the other hand, the unstable regions surrounded by the period-doubling bifurcation points are given by

$$
\beta_{r}<\frac{\omega_{n 2}}{\omega_{n 1}}<\frac{(2 r+1) \pi}{2\left(\pi-\tan ^{-1} \lambda\right)}
$$

where $r$ is a positive integer, and $\alpha_{r}$ and $\beta_{r}$ indicate the values of $\omega_{n 2} / \omega_{n 1}$ satisfying the following equations, respectively:

$$
\alpha_{r}:\left(\frac{\omega_{n 2}}{\omega_{n 1}}\right)=\frac{r \pi-\phi}{\pi-\tan ^{-1} \lambda}, \quad \beta_{r}:\left(\frac{\omega_{n 2}}{\omega_{n 1}}\right)=\frac{(2 r+1) \pi-2 \phi}{2\left(\pi-\tan ^{-1} \lambda\right)} .
$$

The regions denoted by Eqs. (34) and (35) are illustrated in Fig. 7. The regions colored black show the unstable regions based on saddle-node bifurcation [Eq. (34)], and those colored gray show the unstable regions based on period-doubling bifurcation [Eq. (35)]. As $\lambda \rightarrow 0$, saddle-node bifurcation points occur when $\omega_{n 2} / \omega_{n 1} \rightarrow r$, and period-doubling bifurcation points occur when $\omega_{n 2} / \omega_{n 1} \rightarrow r+1 / 2$. These results demonstrate that the unstable regions generated in the in-phase solution branch of the symmetric system are caused by the internal resonance between the first and second linear natural modes. On the other hand, the unstable regions shift to the bigger value of $\omega_{n 2} / \omega_{n 1}$ with increasing $\lambda$ and then widen.

Next, Fig. 8 shows the numerical results obtained using the shooting method for the system with a slight individual difference and the analytical results obtained in this section for the symmetric system. Figure 8(a) shows the same result as in Fig. 3 (the branches generated from bifurcation points are all omitted), and Fig. 8(b) shows the result obtained for $k_{1}=k_{2}=212.0 \mathrm{~N} / \mathrm{m}$ and $c_{1}=c_{2}=C=0.0 \mathrm{~N} \cdot \mathrm{s} / \mathrm{m}$. A slight quantitative difference is observed in the existence region of the stable and unstable solutions due to the individual difference and viscous damping; however, both results agree well. The analytical result for the symmetric system is qualitatively applicable to the system with a slight individual difference. Consequently, it can be regarded that the unstable regions in the Mode 1 branch are caused by internal resonance between the first and second linear natural modes. 


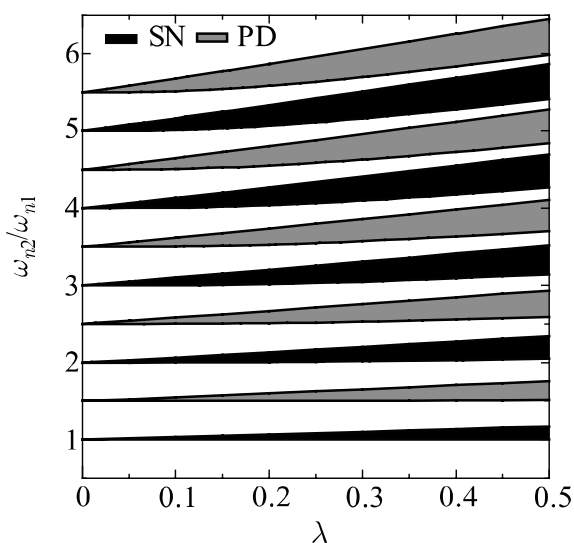

Fig.7 Natural frequency ratio $\omega_{n 2} / \omega_{n 1}$ versus $\lambda$

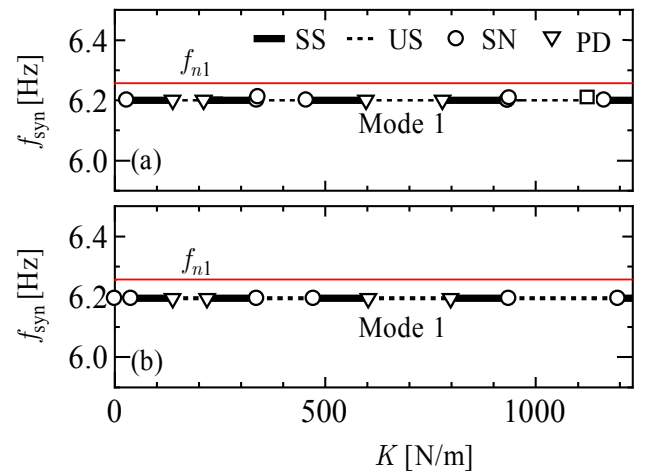

Fig.8 Synchronized solution branch of Mode 1: (a) numerical and (b) analytical results

\section{Conclusion}

As one of the simplest mechanical systems that generate synchronization, a simple model was developed in which two self-excited oscillators with stick-slip motion are directly coupled by a coil spring and a dashpot. The synchronization generated in the present model was investigated both numerically and experimentally. It was proved that two types of stable synchronized solutions with different vibratory patterns exist in the present model, and the characteristics of the synchronized solutions correlate well with the undamped free vibration characteristics of the linear two-degree-of-freedom system in which Coulomb friction was removed. The mechanism behind the synchronization was examined based on the variation in energy transmission between the oscillators through the coupling element and the variation in mechanical energy. As a result, we confirmed that the methods of suitable energy transmission are different according to the individual difference between the oscillators. It was also proved that unstable regions caused by internal resonance exist in the solution branch in which both oscillators vibrate nearly in phase.

In the present model, synchronization occurs only when the individual difference between the oscillators is small, which was not observed in the synchronization generated in previous models ${ }^{(4)-(6),(8)}$. One potential reason is as follows: the stick-slip oscillators that are inferior in their energy adjustment mechanism are directly coupled. As described above, the coupling element assumes important roles in the generation of synchronization. Hence, if the coupling element were improved, synchronization would occur even for a wide range of individual difference. This issue is planned to be discussed in our future work.

\section{Acknowledgements}

The present study was financially supported through a Grant-in-Aid for JSPS Fellows $(22 \cdot 1801)$ by the Japan Society for the Promotion of Science.

\section{References}

(1) A. Pikovsky, M. Rosenblum and J. Kurths, Synchronization (A universal concept in nonlinear sciences), (2001), Cambridge University Press.

(2) J. J. Hopfield, Neurons, dynamics and computation, Physics today, (1994), pp. 40-46.

(3) Nagamine T. and Sato Y., "Self-Synchronization and its application", Transactions of the Japan Society of Mechanical Engineers, Series C, Vol.71, No.704 (2005), pp.1113-1116.

(4) Kondou T., Bonkobara Y., Mori H. and Ishikawa S., Self-synchronized Phenomena Generated in Pendulum-Type Oscillators (1st Report, Analysis for Self-Synchronized Phenomena between Two Metronomes by Using Improved Shooting Method), Transactions of the Japan Society of Mechanical Engineers, Series C, Vol.68, No.676 
(2002), pp.3499-3506.

(5) Bonkobara Y., Mori H., Kondou T. and Ayabe T., Self-Synchronized Phenomena Generated in Rotor-Type Oscillators (1st Report, On the Influence of Coupling Condition between Oscillators), Transactions of the Japan Society of Mechanical Engineers, Series $C$, Vol.73, No.728 (2007), pp.1012-1020.

(6) Mori H., Bonkobara Y., Kondou T. and Ayabe T., Self-Synchronized Phenomena Generated in Rotor-Type Oscillators (2nd Report, Investigation Using Nonlinear Normal Modes), Transactions of the Japan Society of Mechanical Engineers, Series C, Vol.73, No.728 (2007), pp.1021-1028.

(7) The Japan Society of Mechanical Engineers ed., JSME Mechanical Engineers' Handbook, Fundamentals a2 (Dynamics of Machinery), (2004), pp.65-66, The Japan Society of Mechanical Engineers.

(8) Kondou T., Mori H., Bonkobara Y. and Ayabe T., "Moving Apparatus Utilizing Self-Synchronized Phenomena (1st Report, Analysis of Basic Performance by Applying Shooting Method and Experimental Verification), Transactions of the Japan Society of Mechanical Engineers, Series C, Vol.71, No.712 (2005), pp.3351-3358. 